\title{
Effective Algorithm for Detection of Wipes in Presence of Motion and Illumination
}

\author{
S. Chavan ${ }^{1}$ and S. Akojwar ${ }^{2}$ \\ ${ }^{1}$ Department of Electronics and Telecommunication Engg, S.B.J.I.T.M.R Nagpur, M.S \\ ${ }^{2}$ Department of Instrumentation Engineering, Government College of Engineering, Chandrapur-442403, \\ \{Salimsahi197@rediffmail.com ; sudhirakojwar@rediffmail.com\}
}

\begin{abstract}
Shot boundary detection is the first step in the video processing applications. The detection of gradual transition is quite difficult compared to the detection of abrupt transition. Among the different gradual transitions, the detection of wipes is more challenging because the wipes are of different categories and having complex nature. We have presented a novel method for wipe transitions detection. In the proposed algorithm we used Normalized Mean of Approximate Wavelet Co-efficient (NMAWC) as a metric for potential wipe transition detection. To apply algorithm on whole video to detect wipes is computationally expensive. So we tested our algorithm on the video clips containing wipe transitions. The experimental results are evaluated using the performance metrics Recall, Precision, F1 measure, detection rate and the results are compared with the existing methods. Our proposed algorithm achieved a relatively better trade-off between recall and precision as compared to other algorithms. We tested the proposed approach on different wipe effects including Special wipes. The proposed method successfully avoids false positives caused due to object/camera motion and illumination.
\end{abstract}

Keywords: Wipes, NMAWC, Discrete Wavelet Transform, Recall, Precision

\section{Introduction and related work}

Wipe transitions have more complexity and diversity in its transition patterns as compared to cuts, fades and dissolve. Due to this fact research community has given less attention towards detection of wipes and they have concentrated their study on detection of cut, fade and dissolve. In wipe transitions, current shot pixels are replaced by next shot pixels step by step until the current shot is completely replaced by next shot as shown in Fig 1. In such transitions the movement of single or multiple image boundary lines occurs that results into variation in shapes, moving direction and moving speed. There are more than thirty two types of wipe effects commonly used in video editing. These special effect edits are often used in news, sports, cartoons, comedy and show programs. Unlike dissolves and fade, modeling the wipe transition by any single formula is very difficult as their patterns vary considerably. Hence it is a challenging task to detect the presence of all deferent types of wipe transitions as well as to identify their individual patterns.

This work proposes an efficient wipe detection method which can eliminate disturbances caused by noise as well as object motion in detection of wipes. The proposed method works in three stages. In first stage, the features are extracted from the frames using db-6 Discrete Wavelet Transform decomposed to 4 levels. In second stage, normalized mean of approximate wavelet coefficient is calculated for each frame and its variation with frame index detects the potential wipes. In the third stage, color histogram difference is calculated between consecutive frames and from the intersection of two graphs the shot boundaries are declared. The related work for wipe detection in presence of illumination and motion is given below.

A wipe detection method is proposed by U. Naci et al [9] which is based on the fact that two adjacent shots before and after wipes are spatially well separated at any time. The proposed literature addresses the detection of horizontal

B. Iyer, S. Nalbalwar and R. Pawade (Eds.)

ICCASP/ICMMD-2016. Advances in Intelligent Systems Research.

Vol. 137, Pp. 598-609.

(C) 2017- The authors. Published by Atlantis Press

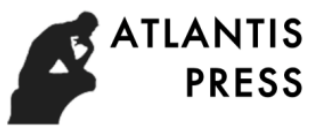

This is an open access article under the CC BY-NC license (http://creativecommons.org/licens)es/by-nc/4.) 
and vertical wipes only. According to wipe detection method proposed by Zabih et al [4], wipes exhibit a characteristic pattern in the ECR time series.

The characteristic pattern can be exploited to detect the potential wipe effects. The proposed algorithm has shown less immunity towards noise and the performance decrease when used over different genres of videos.
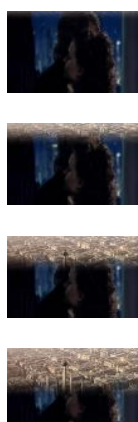
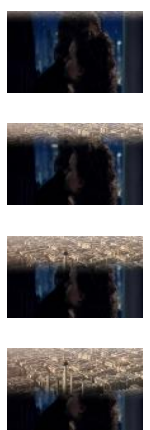
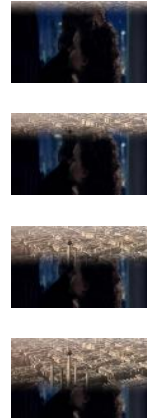
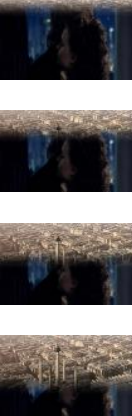
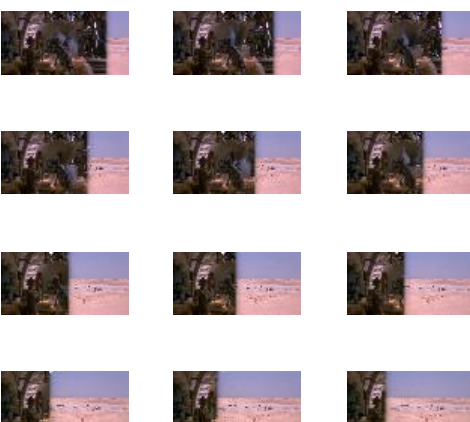

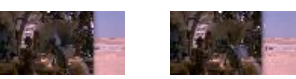

a) Vertical wipe in Star Wars

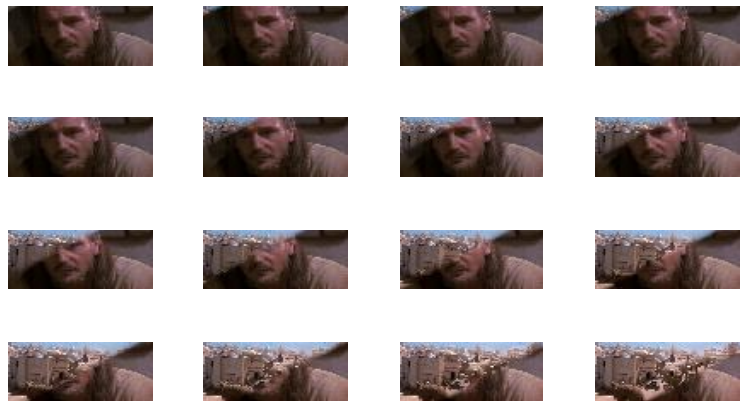

c) Diagonal wipe in Star Wars 1
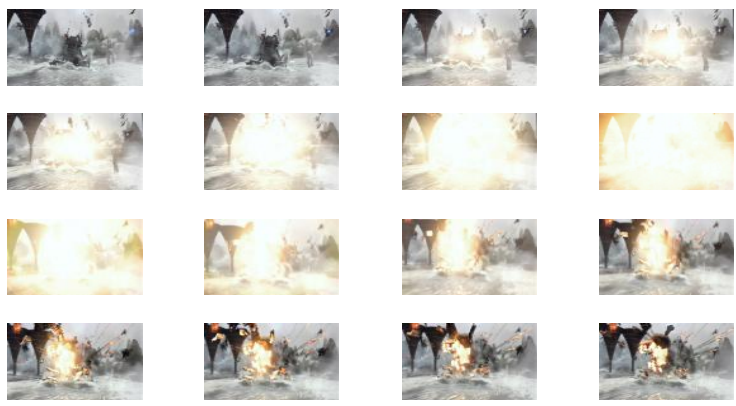

d) Frames containing Lighting effect
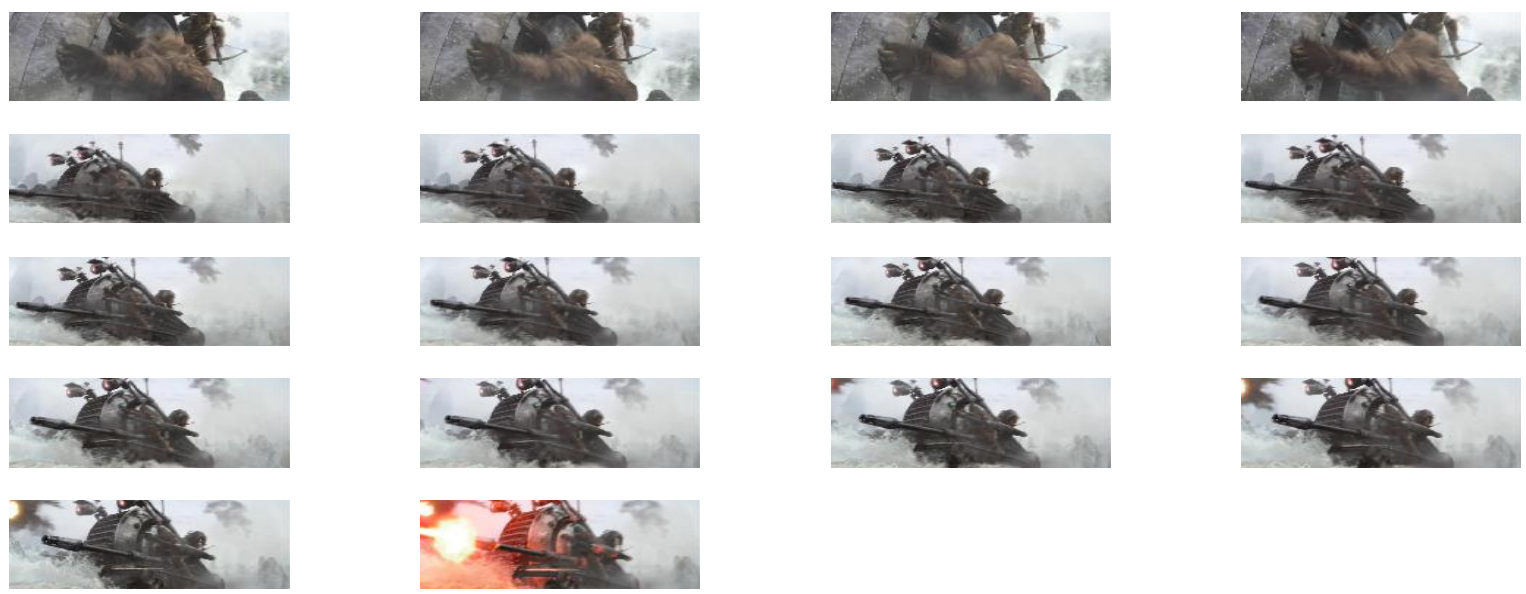

e) Frames containing object motion

Fig 1. various wipe type of transition 
According to a research published by R. Lienhart et al [3], for wipes, the adjacent shots are not temporally separated but spatially well separated at any time. The spatial separation of shots is exploited by the proposed algorithm to effectively detect the potential wipe frames. A wipe detector has been developed by Alattar A. M. et al [10], the proposed wipe detector exploits the linear change in the means and the variances of the frames in the wipe region is developed. Pei et al. [5] have proposed the use of motion vectors to find the scene change regions of each frame. Campisi et al. [6] have developed an algorithm based on the trajectory estimation of the boundary line between two successive frames for wipe detection. Han et al. [7] have used 3D wavelet transform, Gaussian weighted Hausdorff distance, and the direction of motion vector to identify wipes. Mackowiak et al. [8] have employed motion activity, and dominant color descriptors to find wipe region.

A method for wipe detection discriminating object and camera motion is proposed by K. Warhade et al [11]. In the proposed algorithm first the moving strip due to wipe is obtained, which eliminate most of the edges due to object boundaries and retain true wipe boundaries, and then Hough transform is applied on these moving lines to detect and categorize various wipe types. In order to decrease the computational load of the proposed algorithm, the authors propose a preprocessing step as a first stage of the algorithm. The preprocessing step consists of calculation of statistical image difference between the consecutive frames to obtain the potential wipe frames, which are input to their proposed algorithm. The proposed algorithm detects and identifies various types of wipes and also distinguishes wipes from object and camera motion. Fernando W.A.C. et al [1] proposed an approach that taking the benefit of the production feature of video. In the proposed technique, each video frame is first decomposed into low and high-resolution components which are analyzed correspondingly and further recombined together to form a wipe transition detector. In the proposed scheme, the mapping of each image onto the reduced image is done. Then they use structural properties and statistical features of the images to recognize wipe transition region. Finally, Hough transform is used to analyze the wipe patterns and the direction of wipe. The outcome shows that the algorithm is capable of detecting all wipe regions precisely still the video sequence contains other special effects. Nam et al. [2] have used wavelet transform to find direction-emphasized images. These images are projected on a specified angle by Radon transform and then B-Spline interpolation is used to identify wipe region. Mackowiak et al. [8] have employed motion activity and dominant color descriptors to find wipe region.

\section{Proposed method for detection of wipe transitions}

The Proposed Algorithm for detection of wipes is explained as follows.

1. In the proposed algorithm each frame is converted from RGB to Gray level scale.

2. Each frame is decomposed to 4 level using db6 discrete wavelet transform.

3. The mean of approximate wavelet coefficient for each frame is calculated and tabulated in the form of array 'I'.

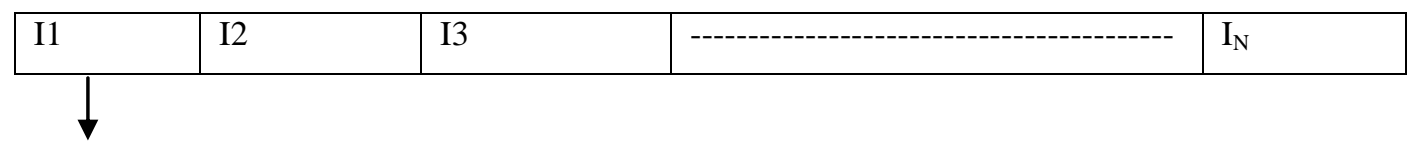

Here, I1 is a mean of wavelet coefficient of first frame.

4. The array ' $\mathrm{I}$ ' is normalized to form an array ' $\mathrm{I}_{\mathrm{N}}$ '. The normalized arra ' $\mathrm{I}_{\mathrm{N}}$ ' is plotted against frame index. For test video the nature of the graph is obtained as Shown in figure 2(a) and (b).

5. From the above sample graphs, it is clear that for all patterns of wipe, there is either a gradual increase or decrease in the graph. Thus this method is capable of detecting almost all types of wipes discriminating the illumination effects very precisely.

6. From the graph, it can be seen that for a wipe the gradual increase or decrease of values will be for more than consecutive 10 frames.

7. The consecutive 10 values of array $\mathrm{I}_{\mathrm{N}}$ which are gradually increasing or decreasing are stored in array ID. 
8. However, in case of camera motion or object motion, we may get a gradual increase or decrease in the graph but of short duration as shown in the graph.

9) To distinguish between camera/object motions and wipe the color histogram difference method is used as post processing technique. In color histogram technique the color histogram is calculated for each frame and it is tabulated in the form of array D.

10) The mean of color histogram is calculated for each frame and it is normalized to form an array $M$ and it is plotted against the frame index as shown in fig. 3

11) Next, the average level of array $\mathrm{M}$ is calculated. The values of array $\mathrm{M}$ which are above average level are stored in an array $\mathrm{N}$.

12) The gradual transition is detected when the values of $M$ are above the average level for more than 10 consecutive frames.

13) The consecutive 10 values of array $\mathrm{M}$ which are above the average level are stored in array Dc.

14) The array $D_{c}$ consists of $n$ numbers of rows. The values of each row are corresponding to gradual transition or camera/object motion. To distinguish between gradual transition and camera/object motion the first and last point of each row are checked, if these values are found to be very close then that row will correspond to gradual transition.

15) To identify only wipes, the intersection of array ID and Dc is calculated. If the intersection of ID and Dc is more than $75 \%$ then profoundly it is cleared that wipe has been detected. As shown in figure 7.4.

16) The types of wipes are also examined in our algorithm by similarity measure.

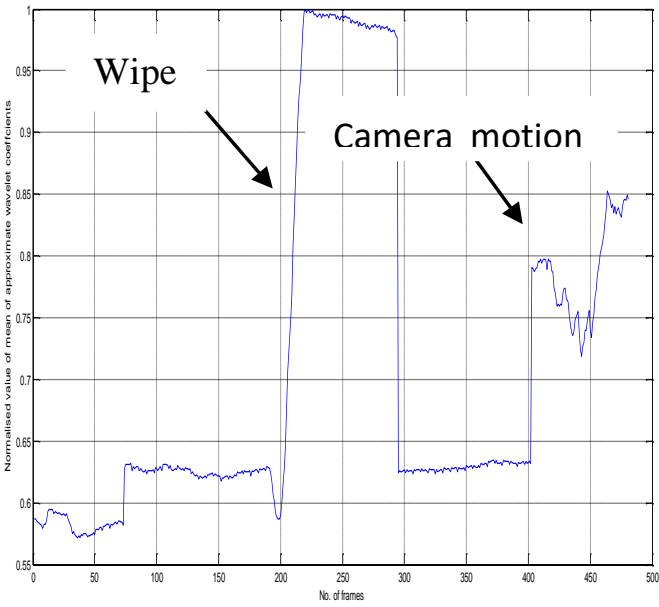

(a)

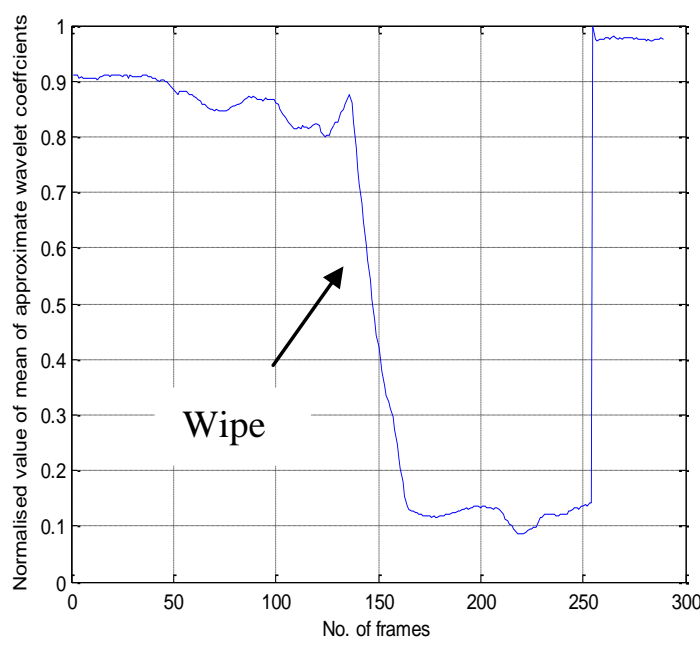

(b)

Fig. 2 (a) and (b) variation in normalized value of mean of approximate wavelet coefficient against frame index

\section{Experimental results and discussion}

\subsection{Test video sequence.}

The proposed algorithm have been tested on various movies, such as Star Wars III, Star Wars I, Jodhaa Akbar and Bhootnath. These movies are manually observed frame by frame for ground truth with the help of Virtual Dub to find the desired detection. In our work, we considered 23 types of wipe patterns broadly categorized into horizontal (denoted by HWP1-HWP7), diagonal (denoted by DWP1-DWP5), vertical (denoted by VWP1-VWP7), and special wipes (denoted by SWP1-SWP4). Number of wipe sequences available in each video with different patterns and information about number of frames with wipe and wipe range is shown in Table 1. To test the effectiveness of the algorithm, we consider the frames from the video where camera and object motion are present before or after wipe. 


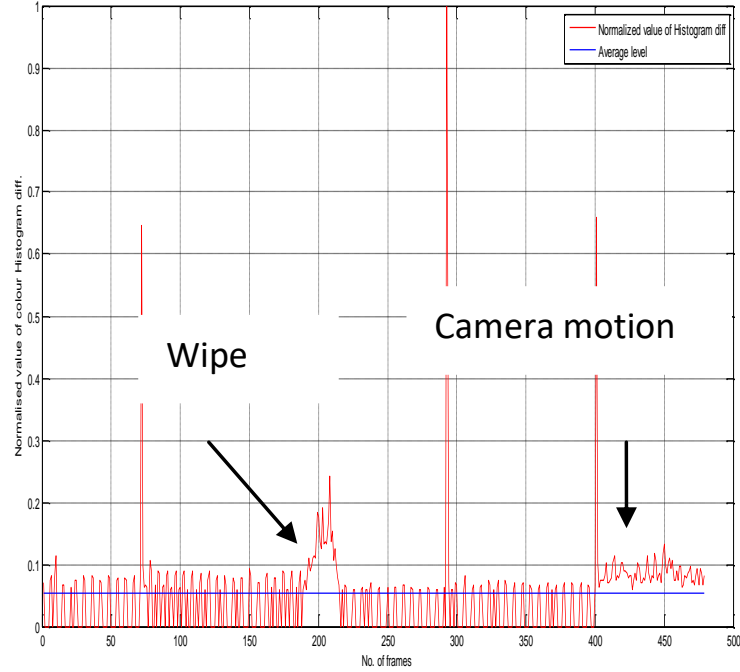

Fig. 3 Variation of Normalized value of color Histogram Difference with frame index

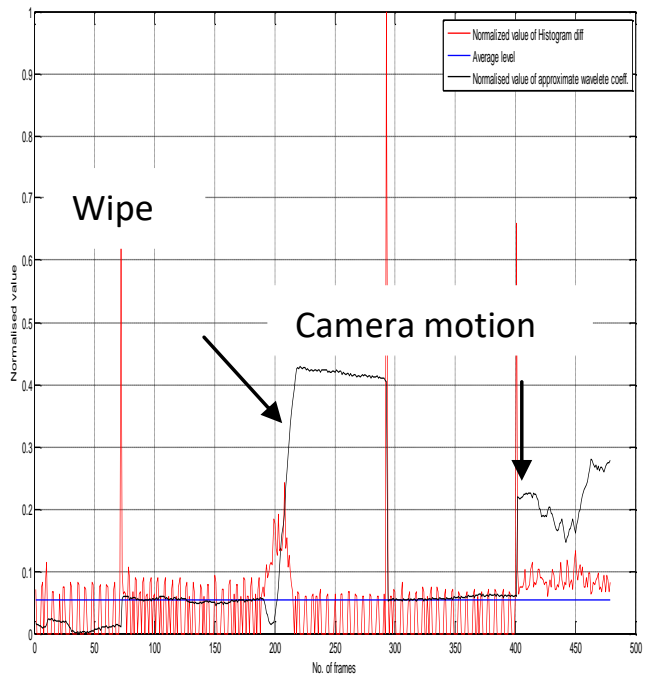

Fig 4 Variation of Normalised values of Mean of Approximate wavelet coefficient and Normalized value of color histogram difference against frame index

\subsection{Evaluation criterion}

We used Recall, Precision and F1 measure as discussed in Section 3.5 as a evaluation metric to compare shot boundary detection algorithm. Generally, wipe transition occurs over a certain range of frames unlike abrupt transition which occurs at a single frame.

In addition to above metrics, we need an additional evaluation metric which can elaborate details about how many wipe types are correctly detected by the algorithm and is given by

Detection Rate $(\mathrm{DR})=\frac{\text { Number of actual wipe types correctly detected }(\text { Hit })}{\text { Number of actual wipe types in the video }(\text { Actual })} \times 100$

Table 1 shows the performance metrics for wipe detection using proposed algorithm. The range of the wipe varies from 12 frames to 60 frames in the test video sequence as shown in Table 1. For the full range of one wipe frames, one wipe type is considered and the number of actual wipe types are found out and shown in Table 2 (Total wipe types movie wise). Any partial detection is considered as correct detection (Hit) if more than 75 percent of its wipe range is correctly declared. Our algorithm is capable of detecting almost all types of wipes with detection rate above $90 \%$. Also it gives good compromise between recall and precision.

We evaluated the performance metrics mentioned above for the videos under test. We have considered the specific movies having lot of rapid camera and object motion and also which are used by other researchers earlier, so that we can compare our algorithm with existing algorithms. Our method is found to be robust in presence of camera, object motion and illumination.

\subsection{Comparison with different wipe detection methods}

Performance of the proposed algorithm has been compared with Alattar [10], Fernando et al. [1], and Nam et al. [2] and K. Warhade [11] for the same video sequences. We selected these algorithms for comparison, since most of the successful methods suggested for wipe detection compared with these algorithms. Above algorithms have been tested for four movie videos, Star Wars III (SWIII), Star Wars I (SWI), Jodhaa Akbar (JA) and Bhootnath( $\mathrm{BN}$ ).Frame based R, P, and F1 measure performance comparison between the proposed method and the other tested algorithms are shown in Table 3. The performance comparison based on detection of wipes (Detection Rate) are shown in Table 2.

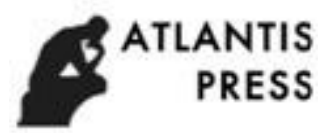


Algorithm by Alattar [10] is based on statistical features and gives false detection for large camera and object motion. As the method is based on statistical features, it detects most of the wipe types but fails in accurately locating the frame range of wipe and results in low Recall but with comparable Detection Rate. As this method does not follow some common pattern, it is difficult to say whether this method will fail or succeed in detecting a particular wipe type. This algorithm is also unable to differentiate between different wipe types like horizontal, vertical, etc. Fernando et al. [1] have used mean square of the image frame difference to find wipe strip.

Table.1 Wipe transition types in each movie

\begin{tabular}{|c|c|c|c|c|c|}
\hline Wipe type & Video & SWIII & SWI & JA & $\mathrm{BN}$ \\
\hline \multirow{8}{*}{ Horizontal Wipe } & HWP1 & 2 & - & - & - \\
\hline & HWP2 & - & - & - & 2 \\
\hline & HWP3 & 3 & - & - & - \\
\hline & HWP4 & - & - & - & 3 \\
\hline & HWP5 & 2 & 12 & 3 & - \\
\hline & HWP6 & 2 & 5 & 5 & - \\
\hline & HWP7 & 3 & - & - & - \\
\hline & Total HW & 12 & 17 & 8 & 5 \\
\hline \multirow{6}{*}{ Diagonal Wipe } & DWP1 & 2 & - & - & - \\
\hline & DWP2 & 2 & - & - & - \\
\hline & DWP3 & 3 & 2 & - & - \\
\hline & DWP4 & 3 & 1 & - & - \\
\hline & DWP5 & 3 & 8 & - & - \\
\hline & Total DW & 13 & 11 & - & - \\
\hline \multirow{8}{*}{ Vertical Wipe } & VWP1 & 1 & - & - & - \\
\hline & VWP2 & 1 & - & - & - \\
\hline & VWP3 & 1 & - & - & - \\
\hline & VWP4 & 1 & - & - & - \\
\hline & VWP5 & 2 & 6 & - & - \\
\hline & VWP6 & 1 & 7 & 1 & - \\
\hline & VWP7 & - & - & - & 2 \\
\hline & Total VW & 8 & 13 & 1 & 2 \\
\hline \multirow{5}{*}{ Special Wipe } & SWP1 & 3 & - & 1 & - \\
\hline & SWP2 & 3 & - & - & - \\
\hline & SWP3 & 2 & - & - & - \\
\hline & SWP4 & 3 & - & - & - \\
\hline & Total SW & 11 & & 1 & \\
\hline Total Wipe Types & Movie wise & 34 & 41 & 10 & 7 \\
\hline Total Wipe Frames & Movie wise & 965 & 1011 & 345 & 105 \\
\hline Frame Range & Min-Max & $9-32$ & $12-30$ & $15-50$ & $10-25$ \\
\hline
\end{tabular}


Table 2 performance metrics for wipe detection

\begin{tabular}{|c|c|c|c|c|c|}
\hline Algorithm & Video & SWIII & SWI & JA & BN \\
\hline \multirow{3}{*}{ Proposed } & R & 91.25 & 91.37 & 97.56 & 89.72 \\
\cline { 2 - 6 } & $\mathrm{P}$ & 95.35 & 99.12 & 92.75 & 89.81 \\
\cline { 2 - 6 } & F1 & 93.25 & 95.08 & 95.09 & 89.76 \\
\hline
\end{tabular}

Table.3 Performance comparison based on detection of wipes

\begin{tabular}{|c|c|c|c|c|c|}
\hline Algorithm & video & SWIII & SW1 & JA & BN \\
\hline \multirow{4}{*}{ Proposed } & Actual & 34 & 41 & 10 & 07 \\
\cline { 2 - 6 } & Hit & 33 & 41 & 09 & 07 \\
\cline { 2 - 6 } & Miss & 01 & 00 & 01 & 00 \\
\cline { 2 - 6 } & DR & 97.06 & 100 & 90 & 100 \\
\hline \multirow{4}{*}{ K. Warhade et at. [11] } & Actual & 34 & 41 & 10 & 07 \\
\cline { 2 - 6 } & Hit & 32 & 41 & 10 & 07 \\
\cline { 2 - 6 } & Miss & 02 & 00 & 00 & 00 \\
\cline { 2 - 6 } & DR & 94.12 & 100 & 100 & 100 \\
\hline \multirow{4}{*}{ Alattar[10] } & Actual & 34 & 41 & 10 & 07 \\
\cline { 2 - 6 } & Hit & 30 & 38 & 09 & 06 \\
\cline { 2 - 6 } & Miss & 04 & 03 & 01 & 01 \\
\cline { 2 - 6 } & DR & 88.24 & 92.68 & 90 & 83.33 \\
\hline \multirow{4}{*}{ Fernando et at. [1] } & Actual & 34 & 41 & 10 & 07 \\
\cline { 2 - 6 } & Hit & 24 & 30 & 09 & 03 \\
\cline { 2 - 6 } & Miss & 10 & 11 & 01 & 04 \\
\cline { 2 - 6 } & DR & 70.59 & 73.17 & 90 & 42.86 \\
\hline \multirow{3}{*}{ Nam et at. [2] } & Actual & 34 & 41 & 10 & 07 \\
\cline { 2 - 6 } & Hit & 29 & 39 & 08 & 04 \\
\cline { 2 - 6 } & Miss & 05 & 02 & 02 & 03 \\
\cline { 2 - 6 } & DR & 85.29 & 95.12 & 80 & 57.14 \\
\hline
\end{tabular}

Table 4 Frame based R, P and F1 measure performance comparison between the proposed and tested algorithms

\begin{tabular}{|c|c|c|c|c|c|}
\hline Algorithm & Video & SWIII & SWI & JA & BN \\
\hline \multirow{3}{*}{ Proposed } & $\mathrm{R}$ & 91.25 & 91.37 & 97.56 & 89.72 \\
\cline { 2 - 5 } & $\mathrm{P}$ & 95.35 & 99.12 & 92.75 & 89.81 \\
\cline { 2 - 5 } & $\mathrm{F} 1$ & 93.25 & 95.08 & 95.09 & 89.76 \\
\hline \multirow{3}{*}{ K.Warhade et al.[11] } & $\mathrm{R}$ & 89.36 & 89.77 & 96.78 & 84.61 \\
\cline { 2 - 5 } & $\mathrm{P}$ & 94.27 & 98.18 & 91.43 & 87.61 \\
\cline { 2 - 5 } & $\mathrm{F} 1$ & 91.74 & 93.78 & 94.02 & 85.97 \\
\hline \multirow{3}{*}{ Alattar[10] } & $\mathrm{R}$ & 65.69 & 73.57 & 79.23 & 74.31 \\
\cline { 2 - 6 } & $\mathrm{P}$ & 70.31 & 79.03 & 86.03 & 48.21 \\
\cline { 2 - 5 } & $\mathrm{F} 1$ & 67.92 & 76.20 & 82.49 & 58.48 \\
\hline
\end{tabular}




\begin{tabular}{|c|c|c|c|c|c|}
\hline \multirow{3}{*}{ Fernando et al.[1] } & $\mathrm{R}$ & 46.19 & 50.09 & 66.37 & 23.85 \\
\cline { 2 - 5 } & $\mathrm{P}$ & 84.70 & 81.55 & 88.32 & 43.33 \\
\cline { 2 - 5 } & $\mathrm{F} 1$ & 59.77 & 62.06 & 75.78 & 30.76 \\
\hline \multirow{3}{*}{ Nam et al.[2] } & $\mathrm{R}$ & 67.67 & 84.53 & 88.88 & 55.04 \\
\cline { 2 - 5 } & $\mathrm{P}$ & 95.44 & 94.93 & 92.12 & 81.08 \\
\cline { 2 - 5 } & $\mathrm{F} 1$ & 79.19 & 89.42 & 90.47 & 60.56 \\
\hline
\end{tabular}

If moving objects are present in the scene in addition to wipe boundary, then binary image shows edges due to wipe as well as edges due to object. Angle of the edge due to wipe and object may be different, and if Hough transform is applied on such frames to find average gradient, it may not give constant angle pattern and results in miss detection. If moving objects are present before or after wipe for long duration, then average gradient may give constant angle pattern and results in false positives. If wipe changes its direction or involves more than two lines, then average gradient pattern may not be constant after applying Hough transform.

This algorithm mainly failed to detect following wipe types: HWP1, HWP7, VWP2, VWP3, VWP4, SWP1, SWP2, SWP3, and SWP4 and results in low Precision and lower Detection Rate. In the algorithm proposed by Nam et al. [2], one of the condition for wipe is that, the wipe transition length must lie between predefined minimum length 15 to maximum length 24 . For a reasonable tradeoff between Recall and Precision, they slightly modified this condition by minimum length 8 to maximum length 58 as per our test video data. This algorithm is unable to detect following wipe types: HWP1, HWP2, VWP1, VWP2, VWP3, and VWP4. This is the main reason for low Recall and comparatively lower Detection Rate in this algorithm. Though this algorithm is not designed and tested for following wipe types by Nam et al. [2]: HWP7, SWP1, SWP2, SWP3, and SWP4, we find that this method is able to detect such wipe types due to its linear like transition behavior and straight line boundaries. This algorithm is unable to locate frame range accurately for this wipe types, but it accurately detects the frame range of other detected wipes and results in higher precision.

The proposed algorithm is able to detect almost all 23 wipe types in this test video sequence and results in high Recall and higher Detection Rate. Previous algorithms failed to detect wipe types VWP3 and VWP4 due to its nonlinear and non rigid changing patterns and boundary. Our algorithm is also able to find out the wipe range accurately in most of the wipe types including HWP7 and diagonal wipes, where scene change region is very small during starting and end of the wipe. This is the main reason for high precision in the proposed algorithm. The proposed algorithm also discriminates wipes from object and camera motion and is demonstrated in the Section 4.

Overall our proposed algorithm gives better trade-off between Recall and Precision as compared to other compared algorithms and detects most of the wipe types and their range accurately. Our proposed algorithm successfully differentiates between horizontal, vertical, diagonal and special wipe types

\section{Demonstration of various wipe patterns obtained using proposed algorithm}

\subsection{Discriminating wipe from object motion and Demonstration of pattern obtained for horizontal wipe}

To show the robustness of the proposed algorithm for discriminating wipe from Object motion, some frames were considered from Star Wars III movie. Ground truth for the above clip is wipe transition is not existing between these frames but the object motion lies from frame 91 to frame 105. Fig 5(a) shows the frames from this video sequence. Output of the proposed algorithm is shown in Fig. 5(b). The results clearly indicate that using the proposed algorithm camera and object motion can be clearly distinguished. 


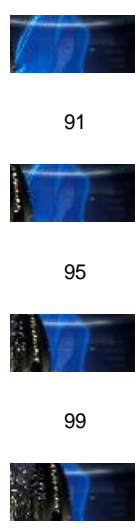

103

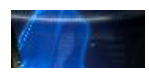

92

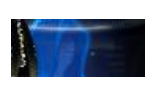

96

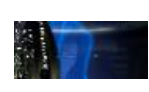

100

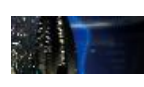

104

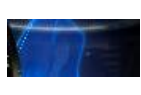

93

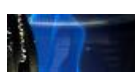

97

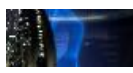

101

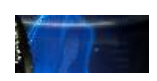

94

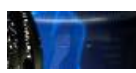

98

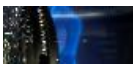

102

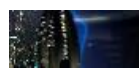

105

(a)

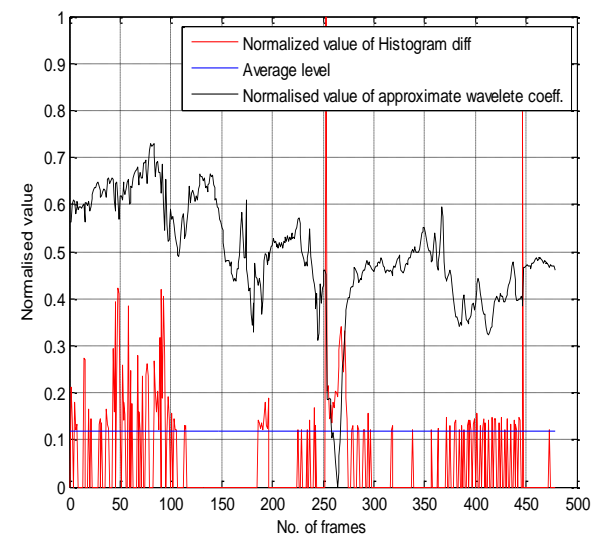

(b)

Fig 5 a) Demonstration of horizontal wipe frames in the movie Star war 3; b) Variation of NMAWC and NCHD with frame index

\subsection{Demonstration of pattern obtained for diagonal wipe}

To demonstrate the results of diagonal wipe, we considered some frames from the movieStar War I. Ground truth for the above clip is wipe transition from 185 to 210 frames. The proposed algorithm is applied on this video clip and result is shown in Fig.6 (a).

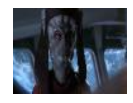

195

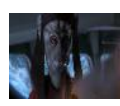

199

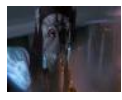

203

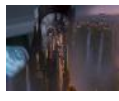

207

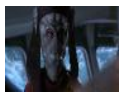

196

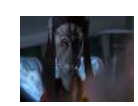

200

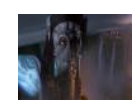

204

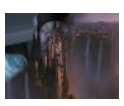

208

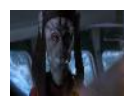

197

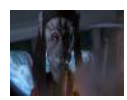

201

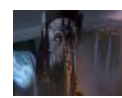

205

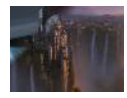

209

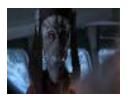

198

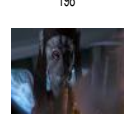

202

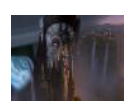

206

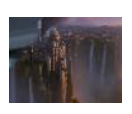

210

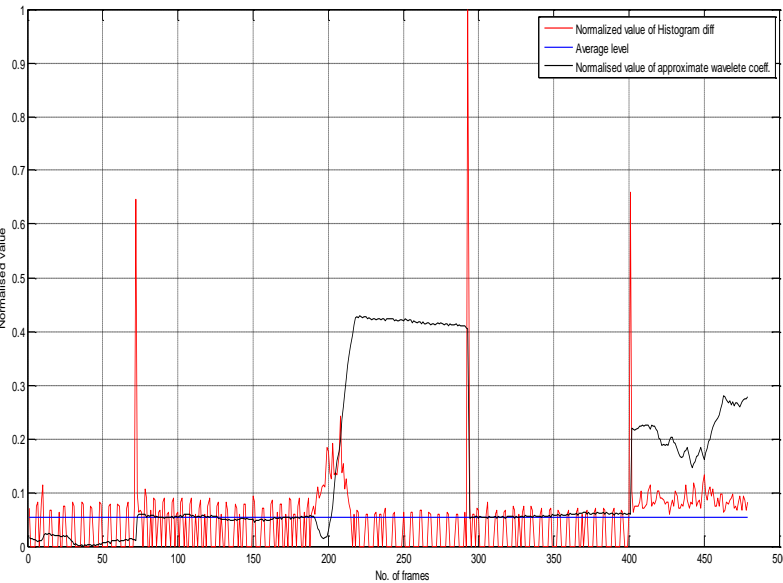

(b)

Fig .6 a) Demonstration of diagonal wipes frames in the movie Star war 1; b) Variation of NMAWC and NCHD with frame index

\subsection{Demonstration of pattern obtained for vertical wipe}

To demonstrate the results of vertical wipe, we considered some frames from the movie Star War I. Ground truth for the above clip is wipe transition from 215 to250. The proposed algorithm is applied on this video clip and result is shown in Fig..7 (a). 


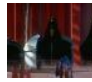

225
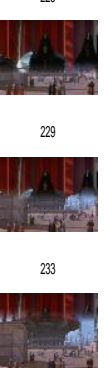

237
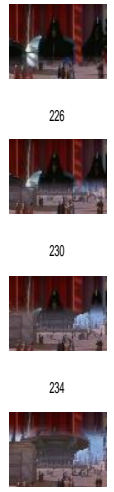

238

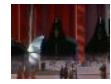

227
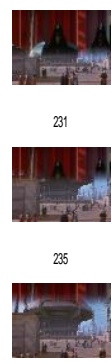

239

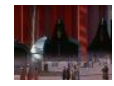

228

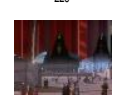

232

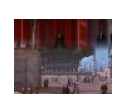

236

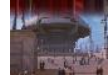

240

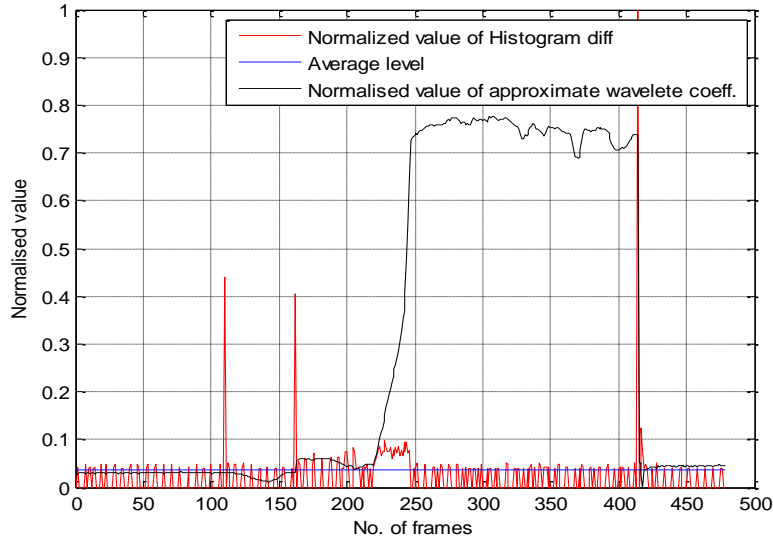

(a)

(b)

Fig 7. a) Demonstration of Vertical wipe frames in the movie Star war 1; b) Variation of NMAWC and NCHD with frame index

\subsection{Demonstration of pattern obtained for special wipe}

To demonstrate the results of this special wipe, we considered some frames from the movie Star War I. Ground truth for the above clip is wipe transition from 225 to 255 . The proposed algorithm is applied on this video clip and result is shown in Fig. 8 (a).
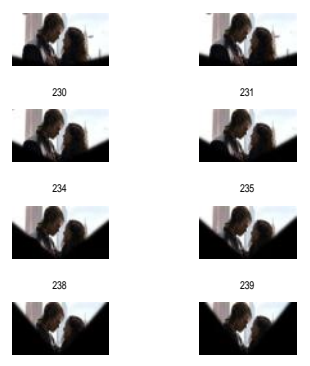

242
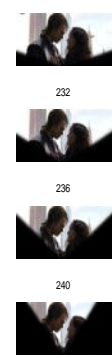

244

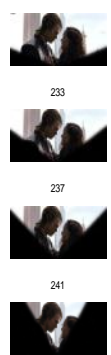

25

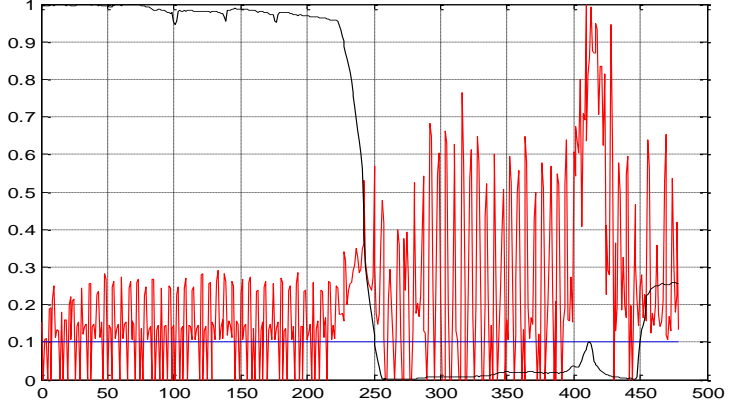

(b)

Fig 8 a) Demonstration of Special wipe frames in the movie Star war 1; b) Variation of NMAWC and NCHD with frame index

\subsection{Discriminating wipe transitions from illumination}

To demonstrate the results of our algorithm in detecting the frames containing wipe in presence of illumination i.e. flash light or explosion, we have considered the video clip of movie star war III.

The ground truth for the above clip is wipe transitions from 236-256. The proposed algorithm is applied on this video clip and the results are presented in figure 9(a) and .9(b).

\section{Summary and conclusions}

In this paper, we propose an algorithm for wipe transition detection. In the proposed algorithm, the presence of wipe is detected by means of discrete wavelet transform and then using color histogram the range and type of wipe can be declared. Applying the proposed algorithm on the whole video to detect wipes is computationally expensive. Our approach can directly be applied for all frames and even then it takes small computational time. 
We extensively tested our proposed algorithm on several genres of video data. Experimental results are carefully evaluated using the performance metrics Recall, Precision, F1 measure, and Detection Rate. Our proposed algorithm achieved a relatively better tradeoff between Recall and Precision as compared to other algorithms. We tested the proposed approach on twenty three different wipe effects and obtained detection Rate of 97.06, 100, 90 and 100 for Star War III, Star War I, Jodhaa Akbar and Bhootnath respectively. The proposed method successfully avoids false positives caused due to object and camera motion. This is the main reason for high precision in the proposed algorithm. The proposed algorithm also discriminates wipes from object and camera motion. Overall our proposed algorithm gave better trade-off between Recall and Precision as compared to other compared algorithms and detected most of the wipe types and their range accurately. Our proposed algorithm successfully differentiated between horizontal, vertical, diagonal and special wipe types.

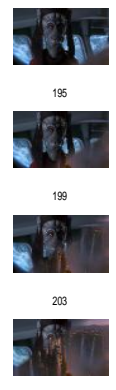

207

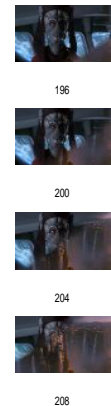

(a)

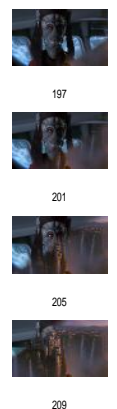

209

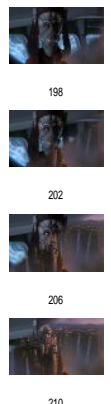

210

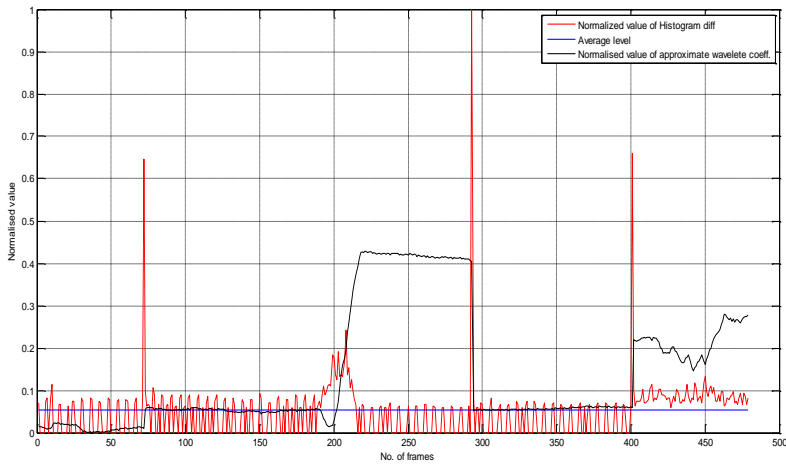

(b)

Fig. .9 (a) Demonstration of wipe frames in the movie star war III containing light effects. (b)Variation of NMAWC and NCHD with frame index

\section{References}

[1] Fernando W. A. C., Canagarajah C. N., and Bull D. R., "Wipe scene change detection in video sequences", Proc. ICASSP, pp. 294-298, 1999.

[2] Nam J. and Tewfik A.H., "Detection of gradual transitions in video sequences using B-Splines interpolation", IEEE Transactions Multimedia, vol. 7, no. 4, pp. 667- 679, 2005.

[3] Lienhart R., "Reliable transition detection in videos: a survey and practitioner's guide", Int. J. Image Graph, vol. 1, no. 3, pp. 469-486, 2001.

[4] Zabih R., Miller J., and Mai K., "A feature-based algorithm for detecting andclassifying scene breaks", Proc. ACM Multimedia, San Francisco, CA, pp. 189-200,

[5] Pei S. C. and Chou Y. Z., "Effective wipe detection in MPEG compressed video using macroblock type information", IEEE Transaction on Multimedia, vol. 4, no. 3, pp. 309-319, 2002.

[6] Campisi P., Neri A., and Sorgi L., "Wipe effect detection for video sequences", IEEE Workshop on Multimedia Signal Processing, pp. 161-164, 2002.

[7] Han B., Ji H., and Gao X., "A 3D wavelet and motion vector based method for wipe transition detection", Proc. ICSP, , no. 2, pp. 1207-1210, 2004.

[8] Mackowiak S. and Relewicz M., "Wipe transition detection based on motion activity and dominant colors descriptors", Proc. 4th Int. Symp. Image Signal Process. Anal., pp. 480- 483, 2005.

[9] U. Naci and A. Hanjalic, "TU Delft at TRECVID 2005: Shot boundary detection," in Proc. TRECVID 2005 Workshop, 2005.

[10] Alattar A.M., "Wipe scene change detector for segmenting uncom-pressed video sequences", ISCAS Proceedings of the 1998 IEEE International Symposium on Volume 4, Circuits and Systems, 1998.

[11] K. Warhade, S.N. Merchant, U.B. Desai, "Effective algorithm for detecting various wipe patterns and discriminating wipe from object and camera motion", Image Processing, IET Volume 4, Issue 6, 2010.

[12] Kwang-deok Seo, Seong Jun Park, and Soon-heung Jung, "Video Partitioning by Temporal Slice Coherency", IEEE Transactions on Consumer Electronics, Vol. 55, No. 2, MAY 2009. 
[13] Zhe Ming Lu, Yongshi, “Fast video shot boundary detection based on SVD and pattern matching”, IEEE Transactions on image processing, vol. 22, No. 12, Dec. 2013.

[14] Chun-Rong Huang, Huai-Ping Lee, and Chu-Song Chen, "Shot Change Detection via Local Keypoint Matching", IEEE Transactions on Multimedia,Vol.10, No. 6, Oct. 2008.

[15] Mallats ,'Theory for multiresolution signal decomposition: The wavelet representation IEEE transaction pattern and mach Intell 1989;11:674-93.

[16] Calvin Chan and Alexander Wong "Shot Boundary Detection using Genetic Algorithm Optimization," IEEE International Symposium on Multimedia 978-0-7695-4589-9/2011.

[17] Zhang xiaona, Qi guoqing, Wang Qiang and Zhang Tao "An Improved Approach of Scene Change Detection in Archived Films" 978-1-4244-5900-1/10@2010 IEEE

[18] Joe Diggins, "Detecting Cross-Fades in Interlaced Video with 3:2 Film Cadence," IEEE transactions on circuits and systems for video technology, vol. 19, no. 7, July 20091063 